\title{
A New Electronic Detector for FIM and AP: Initial WoW Results
}

\author{
P.P. Camus*,**, D.J. Larson*,***, L.M. Holzman*** and T.F. Kelly*,**,*** \\ * Applied Superconductivity Center, University of Wisconsin, Madison, WI 53706, U.S.A. \\ ** Department of Materials Science and Engineering, University of Wisconsin, Madison, WI 53706, \\ U.S.A. \\ *** Materials Science Program, University of Wisconsin, Madison, WI 53706, U.S.A.
}

\begin{abstract}
A new style of charge-separation position-sensitive detector has been tested which has similar cost benefits as other charge-separation devices but has an error-awareness capability to provide an increased collection rate. The wedge-on-wedge anode is similar to the wedge-and-strip anode except that the four electrodes possess both wedge and strip character. This provides additional charge information such that a plurality of positional equations occurs. This provides an error-awareness condition if multiple arrivals strike the detector. Initial results of the wedge-on-wedge detector for use as an electronic detector for imaging and analysis for atom probe microscopy are provided. These results indicate that this detector should perform adequately for atom probe microscopy.
\end{abstract}

\section{BACKGROUND}

Many imaging and analytical techniques require the use of position-sensitive detectors (PSD) for data collection. Several classes of PSDs use the charge cloud produced by microchannel plates (MCP) for position determinations [1]. These classes can be broadly described as discrete anode, charge separation, and hybrid. Discrete anode detectors require that the entire charge cloud land on a single anode. This produces a binary arrival event at the anode location in the discrete anode array. Charge-separation devices require that the charge be divided among several electrodes. The ratio of these charges provides two-dimensional (2D) positioning information. Hybrid systems require that the charge cloud land on multiple anodes such that coarse positioning is determined by the anode location and fine positioning is determined by charge-separation means.

The advantage of discrete anodes is the parallel detection of incoming events. Ideal parallel detection provides the highest encoding rate and lowest error rate thus providing the greatest valid data collection rate. A disadvantage of discrete anode detectors is the large number of anodes and electronic circuits required in the ideal case ( $n$ by $n$, where $n \sim 500$ ). Schemes have been determined to reduce the number of electronic circuits, but cost is still an issue.

An advantage of charge separation devices is the limited number of electronics circuits $(\sim 4)$ required for a large number of pixels $(\mathrm{n} \sim 500)$. This keeps hardware costs manageable while providing for good imaging performance. However, the limited number of circuits also leads to the disadvantage that these detectors can only process events serially, i.e., they are only single-event detectors. This means that only a single event can be processed within the detector cycle time (collection and measurement). The incoming event rate should be sufficiently low so that the probability of two incoming events during a single cycle is lower than the tolerable error rate.

Hybrid systems adopt the advantages of each of these systems without encountering their disadvantages. High valid data encoding rates are possible through the use of parallel detection and electronics but hardware savings are encountered through the enhanced resolution of the reduced number of circuits by using charge-separation schemes. 
Charge-separation (followed by hybrid) schemes are the most widely used in single-particle PSD applications (astrophysics and scientific imaging). Resistive anode (RA) detectors were prevalent, however non-linearity and thermal charge noise [2] restricted their performance. Wedge-and-strip anode (WSA) detectors contain 3 or 4 geometrically-shaped electrodes and avoid the performance problems of RA detectors while increasing the resultant image resolution [1]. Because of these considerations, hybrid mosaic WSA detectors have been produced which provide some parallel detection for both increased encoding rates and higher resolution [3]. Encoding problems at electrode boundaries was also discussed and resolved.

The main shortcoming of the charge-separation class of detectors is the impossibility of the detection of the arrival of multiple events by the anode during a single detector cycle. The operation of the whole detector assembly can be optimized to provide partial multiple event detection. The calculated position of multiple arrivals is the weighted-mean position of all of the charge clouds, which provides erroneous data. All single-event detectors require that the average number of events per cycle be kept quite low. For example, when randomly arriving events follow Poisson statistics, an average arrival rate of about 1 in 100 detector cycles is required to achieve an error rate less than 1 in $10^{4}$. In the specific case of AP analyses, the incoming rate is not Poisson in nature but correlated, which places even harsher restrictions on reducing error rate by decreasing the incoming rate. To maintain a given error rate while increasing the valid encoding rate, the detector cycle time must be reduced or multi-hit capabilities must be used. An alternative to maintaining a low error rate while increasing the encoding rate would be the ability to discern and reject erroneous data. This "error awareness" could lead to up to an order of magnitude increase in the valid encoding rate for such a detector. Multiple-hit encoding (parallelism) or multi-hit awareness are key features for increasing the encoding efficiency of this class of detectors.

PSDs have been used for both imaging and analysis for atom probe field ion microscopy (APFIM) applications. A RA detector was used to obtain digital FIM and desorption images at sufficient resolution and speed for specimen analysis [4]. A WSA has been successfully used in conjunction with time-of-flight (TOF) information to provide the first 3D images of the arrangements of atoms within alloys [5]. A hybrid discrete anode system provides much the same information, but at a higher encoding rate and with more electronics [6]

The need still exists for a less complicated and inexpensive PSD scheme which can correctly encode incoming events at a high flux. A detector that has error awareness of multiple events could increase the data encoding rate by an order of magnitude at the same error rate or produce a lower error rate at the same arrival rate. In an effort to address these concerns, an innovative charge separation detector was recently conceived and implemented that is based on similar principles as the WSA but incorporates a method to detect and reject multiple arrival events within a detector cycle. The wedge-onwedge (WoW) detector was designed with electronic redundancy to provide this error awareness capability.

The WoW has four geometric electrodes which vary in size in both the $\mathrm{x}$ and $\mathrm{y}$ directions [7-9], Figure 1. This provides redundancy in the calculated arrival positions. If the redundant equations do not provide consistent values, multiple arrivals are detected and an error condition is sensed. This data can be removed from the data set reducing the noise in the image. The error awareness only works if all of the events are separated by a threshold distance in both the $\mathrm{x}$ and $\mathrm{y}$ directions.

Preliminary results using this detector for both imaging and analysis applications useful for atom probe microscopy follows.

\section{EXPERIMENTAL}

The base instrument for current the detector testing is the Atom Probe Microscope built at the University of Wisconsin-Madison. It started life as a NORAN Instruments ADEM SEM but has been retrofitted to provide APFIM capabilities. These include enhanced vacuum, stage connections of high voltage and cryogenic cooling, image gas handling, MCP-phosphor screen assembly, and time-of-flight analysis using voltage pulsing [10].

Two different WoW detectors were tested: a single coplanar anode and a 2-by-2 mosaic of coplanar anodes. The coplanar construction requires that all four electrodes are "serpentine" in geometry. The single anode detector was $38 \mathrm{~mm}$ square and the mosaic was $43 \mathrm{~mm}$ square. Each anode is mounted behind a $75 \mathrm{~mm}$ chevron MCP assembly. The anode charges were amplified by $20 \mathrm{x}$ before being measured by a charge-to-digital-converter (QDC). A signal from the MCP was used to set the gate duration for the QDC collection window (10-150ns range, 50-70ns typical). The charge values were read from the CAMAC module to a microcomputer for the calculation of positional information. If TOF information 
was required, the timing stop signals were sent from the MCP to the time-to-digital converter (TDC). The measured times were saved synchronically with the position data. Analysis routines were implemented to translate the raw data (individual electrode charges and TOF) into the required format for display ( $x, y, z$, mass-to-charge).

\section{RESULTS}

Unfortunately, a physical defect was found on the single WoW which prevented collection of charge from one electrode. This meant that only 3 charges could be measured and used for image formation. This limitation removed the error-awareness capability of this detector, limiting it to the same capabilities as that of a WSA detector. Positioning capability with this anode was only slightly compromised by the apparent charging of the defective electrode in one corner and the resultant distortion of the arrival positions in this portion of the detector.

The shapes of the pulses coming from both the MCP and the electrodes were investigated to determine the necessary length of the integration window for the QDC. The signal from the MCP has the expected $\sim 3$ ns wide FWHM RC pulse shape while that from the electrodes was not qualitatively different in shape, only slightly spread in time $(\sim 5 \mathrm{~ns}$ FWHM $)$, Figure 2 . Impedance mismatching was observed on the electrode signals by the appearance of ringing of the signal for times up to $\sim 40-50 \mathrm{~ns}$. To reduce the effect of this ringing on the charge collection, the end of the integration window of the QDC was chosen as the time that the anode signal reached baseline, $\sim 70 \mathrm{~ns}$ after the window start. If impedance mismatching is reduced in the circuit, a shorter integration window should be possible.

When these electrode signals were amplified by $200 \mathrm{x}$, the amplitude exceeded the maximum voltage of the preamplifiers which then truncated the signals. When the signals were amplified by $20 \mathrm{x}$, no signals were truncated, however the full charge range of the QDC was not utilized. This limitation hinders the ultimate performance of the detector and will be rectified in the next upgrade.

A mask containing four circular holes was placed in front of the single-anode detector for performance testing. A nearly uniform intensity from field ionized imaging gas was used to illuminate the mask and obtain an image, Figure 3 . The image resolution was determined from this image by simulating the broadening of the mask image by convolution with a Gaussian. The standard deviation of the Gaussian that produces the same $10 \%$-intensity image width provides the half-resolution value. This value gives an image resolution of $38 \mu \mathrm{m}$ or $\sim 850$ image pixels.

To eliminate the phosphor screen and produce a fully electronic detector system, the WoW must have the ability to collect an electronic image. An electronic FIM image, Figure 4, was collected at an image gas pressure much reduced $\left(1 \times 10^{-5} \mathrm{~Pa}\right)$ from that used for imaging with a typical phosphor detector. This reduction is required to reduce the imaging gas ionization rate and thus the number of multiple arrivals [5]. Although data from multi-hits could not be removed while forming the image obtained from the single anode, features previously observed in the FIM image, Figure 5, were recognized and correlated well with those in the electronic image.

A test was performed to determine the maximum rate of data collection and encoding from the single anode detector. A "uniform" ion source (not a FIM specimen) was used to fully illuminate the detector. When the charge data was printed to the computer screen during collection, the maximum incoming rate from the single anode detector was computer limited to $\sim 400$ ions per second. When this visual record was not provided, but the data was stored on the computer, the maximum rate was increased to $\sim 1000$ ions per second. It is surmised that the speed restriction is between the computer and the QDC and higher rates should be possible with dedicated electronics.

For use as an AP detector, the positional information of the incoming ions must be correlated with the TOF of those ions. A series of metals were analyzed in TOF mode using the WoW to illustrate the applicability of the detector for materials analyses. It was found that the resolution of the mass spectra were not degraded by the use of the timing signal from the MCP compared to using the signal from a phosphor screen. The evaporation rate was kept low when using the defective single-anode detector to reduce the probability of multiple-hit arrivals. If multi-hits were determined from the TDC, that data was not included in the image. Tungsten was analyzed because of the wide separation in mass between the various charged species $(+2$ through +4$)$. Nb-46wt. \% Ti was also analyzed, however no alpha-Ti precipitates were observed.

A specimen of 304 stainless steel was also analyzed in the 3DAP. Because the mass resolution was comparable to that of other short flight-path instruments, selection of the mass ranges was difficult for the closely spaced transition metal peaks of $\mathrm{Cr}, \mathrm{Fe}$, and $\mathrm{Ni}$. Even so, ranges could be selected and a 3D image produced, Figure 6 . The depth scale was calibrated by determining the average number of ions 
per plane using the average material density and expected detection efficiency of the MCPs. In this image, the left side appears to have a higher concentration of $\mathrm{Ni}$ while the $\mathrm{Cr}$ seems to be uniformly distributed. The concentration of $\mathrm{Ni}$ suggests the presence of a phase boundary, which was also observed in the FIM image. A slight amount of $\mathrm{O}$ was also detected in the analysis.

\section{CONCLUSIONS}

A new style of charge-separation position-sensitive detector has been built and tested. The WoW detector has an intrinsic error-awareness ability for the removal of erroneously positioned multi-hit arrivals. It can be used both for electronic imaging and materials analysis applications in conjunction with TOF. This capability makes it preferable for application that previously used WSA detectors.

\section{References}

[1] J. G. Timothy, Photoelectronic Image Devices 1991, (Institute of Physics Publishing Ltd., Bristol, England, 1992) 85.

[2] C. Martin, P. Jelinsky, M. Lampton, and R. F. Malina, Rev. Sci. Instrum. 52 (1981) 1067.

[3] C. Martin and A. Rasmussen, IEEE Trans. On Nuclear Sci. 36 (1989) 836.

[4] T. Schiller, U. Weigmann, S. Jaenicke, and J. H. Block, Jnl. de Phys. Colloq C2 (1986) 479.

[5] A. Cerezo, T. J. Godfrey, and G. D. W. Smith, Rev. Sci. Instrum. 59 (1988) 862.

[6] B. Deconihout, A. Bostel, P. Bas, S. Chambreland, L. Letellier, F. Danoix, and D. Blavette, Appl. Surf. Sci. 76/77 (1994) 145.

[7] L. M. Holzman, T. F. Kelly, and P. P. Camus, US Patent \# 5,347,132 (Sept. 13, 1994).

[8] L. M. Holzman, P. P. Camus, and T. F. Kelly, Proc. of the $40^{\text {th }}$ International Field Emission Symposium (Nagoya, Japan, 1993) 31.

[9] T. F. Kelly, P. P. Camus, L. M. Holzman, and D. J. Larson, To be submitted to Rev. Sci. Instrum.

[10] D. J. Larson, Ph. D. Thesis, University of Wisconsin (1996).

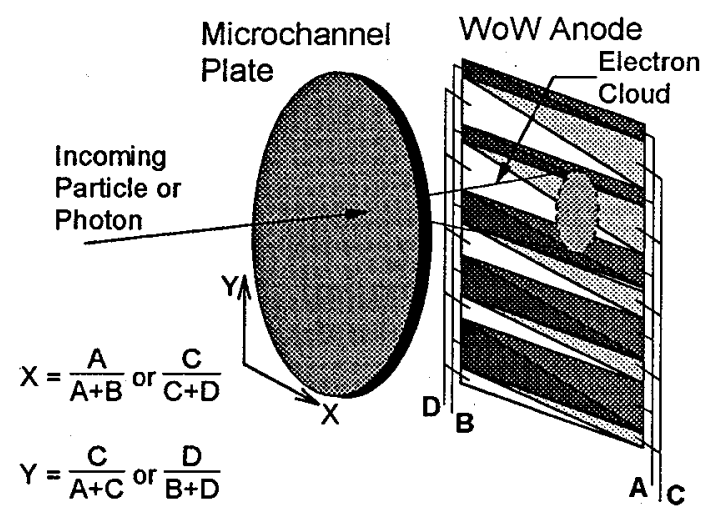

Figure 1 Schematic diagram of the positioning nature of the WoW detector.

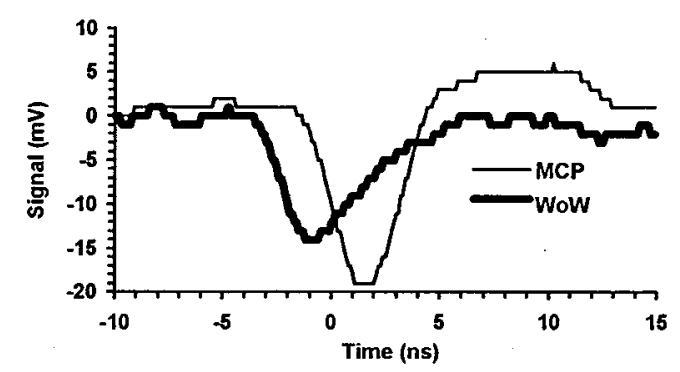

Figure 2 Unamplified pulses from a chevron MCP (inverted signal) and a single electrode of the WoW using a $200 \mathrm{MHz}$ oscilloscope.

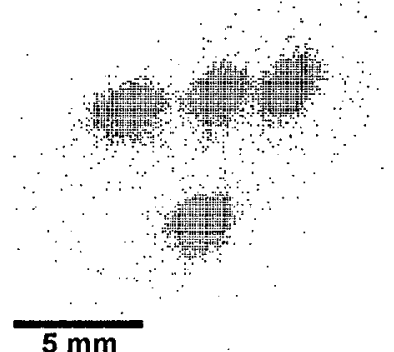

Figure 3 Image obtained through a 4-hole mask from the WoW detector. 


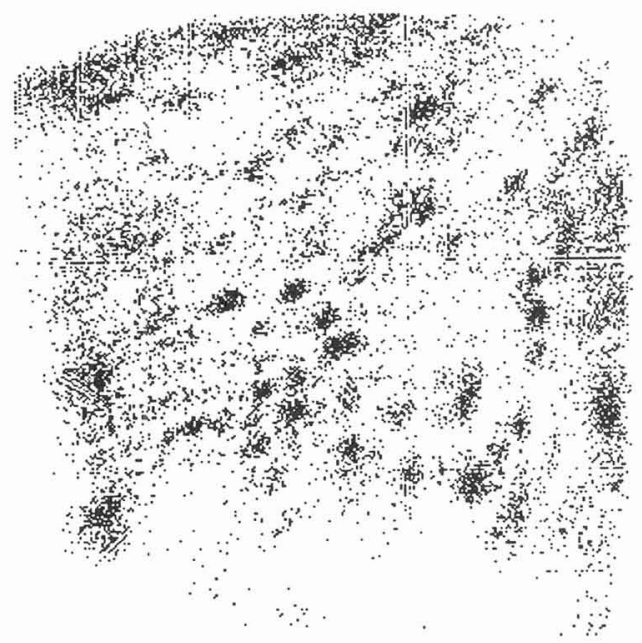

Figure 4 Electronic FIM collected on the single anode WoW detector. Individual atoms can be resolved in the image.

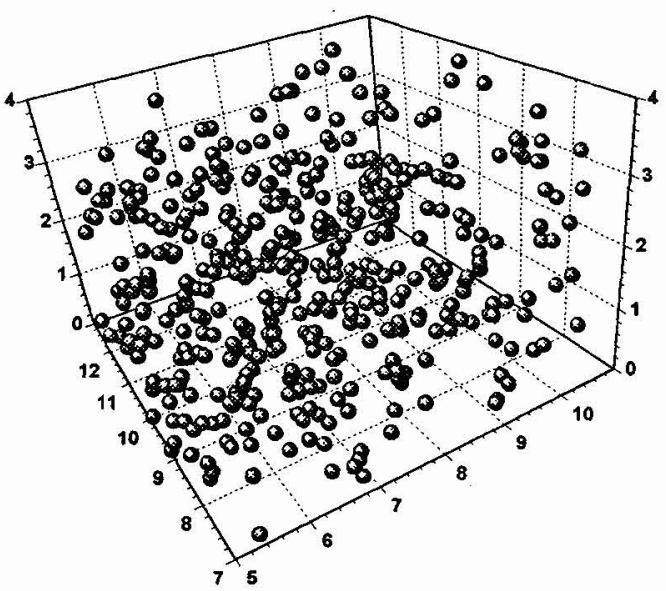

(a)

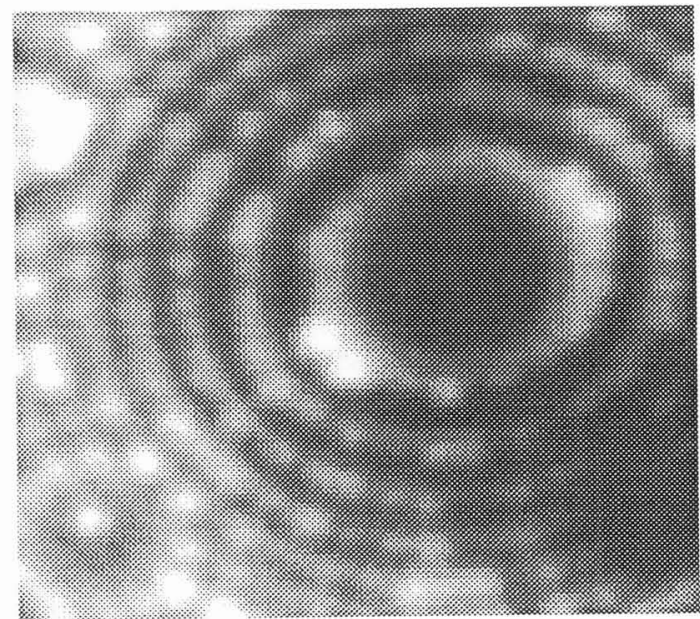

Figure 5 FIM image taken from the phosphor screen imaging detector cropped to a similar size and orientation as Fig. 4.

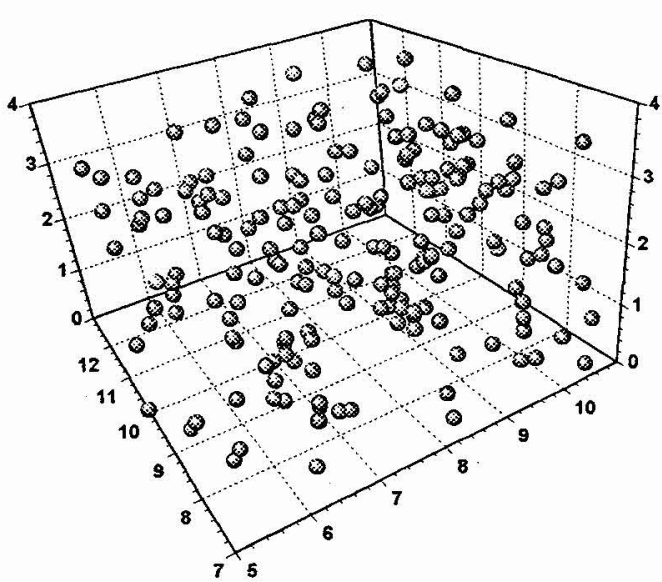

(b)

Figure 6 A subset 3DAP image of the analysis of 304 stainless steel: a) nickel ions and b) cliromium ions. Each shaded ball represents an ion of the given element positioned at its measured location within the analysis volume. The predominant $\mathrm{Fe}$ ions have been removed for clarity. All dimensions in $\mathrm{nm}$.

\section{Acknowledgments}

The authors acknowledge W. Starch for assistance with fabrication of the detector, D. L. Preston for specimen preparation of $\mathrm{Nb}$-Ti and Dr. S. S. Bajikar for helpful discussions. This research was supported by the National Science Foundation, Division of Materials Research under grant \#DMR-8911332. 